\title{
Identification of prognostic risk factors for pancreatic cancer using bioinformatics analysis
}

Dandan Jin ${ }^{1,2}$, Yujie Jiao ${ }^{1,2}$, Jie Ji ${ }^{1,2}$, Wei Jiang ${ }^{3}$, Wenkai $\mathrm{Ni}^{1}$, Yingcheng $\mathbf{W u}^{2}$, Runzhou $\mathbf{N i}^{1}$, Cuihua Lu ${ }^{1}$, Lishuai $\mathrm{Qu}^{1}$, Hongbing $\mathrm{Ni}^{1}{ }^{1}$, Jinxia Liu ${ }^{1}$, Weisong $\mathrm{Xu}^{\text {Corresp., } 4}$, MingBing Xiao ${ }^{\text {Corresp. } 1,5}$

${ }^{1}$ Department of Gastroenterology, Affiliated Hospital of Nantong University, Nantong, China

2 Clinical Medicine, Medical College, Nantong University, Nantong, China

3 Department of Emergency, Affiliated Hospital of Nantong University, Nantong, China

4 Department of Gastroenterology, Second People's Hospital of Nantong, Nantong, China

5 Research Center of Clinical Medicine, Affiliated Hospital of Nantong University, Nantong, China

Corresponding Authors: Weisong Xu, MingBing Xiao

Email address: xws71@sina.com, xmb73@163.com

Background: Pancreatic cancer is one of the most common malignant cancers worldwide. Currently, the pathogenesis of pancreatic cancer remains unclear; thus, it is necessary to explore its precise molecular mechanisms. Methods: To identify candidate genes involved in the tumorigenesis and proliferation of pancreatic cancer, the microarray datasets GSE32676, GSE15471 and GSE71989 were downloaded from the Gene Expression Omnibus (GEO) database. Differentially expressed genes (DEGs) between Pancreatic ductal adenocarcinoma (PDAC) and nonmalignant samples were screened by GEO2R. The Database for Annotation Visualization and Integrated Discovery (DAVID) online tool was used to obtain a synthetic set of functional annotation information for the DEGs. A PPI network of the DEGs was established using the Search Tool for the Retrieval of Interacting Genes (STRING) database, and a combination of more than 0.4 was considered statistically significant for the PPI. Subsequently, we visualized the PPI network using Cytoscape. Functional module analysis was then performed using Molecular Complex Detection (MCODE). Genes with a degree $\geq 10$ were chosen as hub genes, and pathways of the hub genes were visualized using ClueGO and CluePedia. Additionally, GenCLiP 2.0 was used to explore interactions of hub genes. The Literature Mining Gene Networks module was applied to explore the cocitation of hub genes. The Cytoscape plugin iRegulon was employed to analyze transcription factors regulating the hub genes. Furthermore, the expression levels of the 13 hub genes in pancreatic cancer tissues and normal samples were validated using the Gene Expression Profiling Interactive Analysis (GEPIA) platform. Moreover, overall survival and disease-free survival analyses according to the expression of hub genes were performed using Kaplan-Meier curve analysis in the cBioPortal online platform. The relationship between expression level and tumor grade was analyzed using 
the online database Oncomine. Lastly, the eight snap-frozen tumorous and adjacent noncancerous adjacent tissues of pancreatic cancer patients used to detect the CDK1 and CEP55 protein levels by western blot. Conclusions: Altogether, the DEGs and hub genes identified in this work can help uncover the molecular mechanisms underlying the tumorigenesis of pancreatic cancer and provide potential targets for the diagnosis and treatment of this disease. 


\section{Identification of prognostic risk factors for pancreatic cancer using}

\section{2 bioinformatics analysis}

3 Dandan Jin ${ }^{1,3}$, Yujie Jiao ${ }^{1,3}$, Jie $\mathrm{Ji}^{1,3}$, Wei Jiang ${ }^{4}$, Wenkai $\mathrm{Ni}^{1}$, Yingcheng $\mathrm{Wu}^{3}$, Runzhou $\mathrm{Ni}^{1}$, Cuihua $\mathrm{Lu}^{1}$,

4 Lishuai Qu ${ }^{1}$, Hongbing Ni ${ }^{1}$, Jinxia Liu ${ }^{1}$, Weisong $\mathrm{Xu}^{5, *}$, Mingbing Xiao ${ }^{1,2, *}$

$5 \quad{ }^{1}$ Department of Gastroenterology, Affiliated Hospital of Nantong University, Nantong, China

$6 \quad{ }^{2}$ Research Center of Clinical Medicine, Affiliated Hospital of Nantong University, Nantong, China

$7 \quad{ }^{3}$ Clinical Medicine, Medical College, Nantong University, Nantong, China

$8{ }^{4}$ Department of Emergency, Affiliated Hospital of Nantong University, Nantong, China

$9{ }^{5}$ Department of Gastroenterology, Second People's Hospital of Nantong, Nantong, China

$10 \square$ The First Corresponding autho

11 Mingbing Xiao

12 xmb73@163.com (M.Xiao).

$13 \square$ The second Corresponding author

14 Weisong Xu

15 ws71@sina.com (W.Xu). 


\section{Abstract}

32

Background: Pancreatic cancer is one of the most common malignant cancers worldwide. Currently, the pathogenesis of pancreatic cancer remains unclear; thus, it is necessary to explore its precise molecular mechanisms.

Methods: To identify candidate genes involved in the tumorigenesis and proliferation of pancreatic cancer, the microarray datasets GSE32676, GSE15471 and GSE71989 were downloaded from the Gene Expression Omnibus (GEO) database. Differentially expressed genes (DEGs) between Pancreatic ductal adenocarcinoma (PDAC) and nonmalignant samples were screened by GEO2R. The Database for Annotation Visualization and Integrated Discovery (DAVID) online tool was used to obtain a synthetic set of functional annotation information for the DEGs. A PPI network of the DEGs was established using the Search Tool for the Retrieval of Interacting Genes (STRING) database, and a combination of more than 0.4 was considered statistically significant for the PPI. Subsequently, we visualized the PPI network using Cytoscape. Functional module analysis was then performed using Molecular Complex Detection (MCODE). Genes with a degree $\geqslant 10$ were chosen as hub genes, and pathways of the hub genes were visualized using ClueGO and CluePedia. Additionally, GenCLiP 2.0 was used to explore interactions of hub genes. The Literature Mining Gene Networks module was applied to explore the cocitation of hub genes. The Cytoscape plugin iRegulon was employed to analyze transcription factors regulating the hub genes. Furthermore, the expression levels of the 13 hub genes in pancreatic cancer tissues and normal samples were validated using the Gene Expression Profiling Interactive Analysis (GEPIA) platform. Moreover, overall survival and disease-free survival analyses according to the expression of hub genes were performed using Kaplan-Meier curve analysis in the cBioPortal online platform. The relationship between expression level and tumor grade was analyzed using the online database Oncomine. Lastly, the eight snap-frozen tumorous and adjacent noncancerous adjacent tissues of pancreatic cancer patients used to detect the CDK1 and CEP55 protein levels by western blot. 
54 Conclusions: Altogether, the DEGs and hub genes identified in this work can help uncover the molecular

55 mechanisms underlying the tumorigenesis of pancreatic cancer and provide potential targets for the diagnosis and treatment of this disease.

\section{Introduction}

Pancreatic cancer is one of the most common lethal tumors worldwide, and its overall 5-year survival rate is less than 5\% in the United States. PDAC accounts for 95\% of all pancreatic cancers (Siegel et al. 2018). Moreover, it has been reported that only $10-15 \%$ of pancreatic cancer patients are eligible for tumor resection, a trend that is attributed to the lack of early diagnostic markers and advanced metastasis (Becker et al. 2016; Caruso Bavisotto et al. 2017). Mounting evidence indicates that abnormal expression and gene variants are related to the tumorigenesis and progression of pancreatic cancer. A study by Caldas C showed that K-ras activation was involved in early events in N-nitroso-bis (2-oxopropyl) amine-induced pancreatic carcinogenesis in hamsters (Caldas et al. 1994). In addition, it has been reported that inactivation of SMAD family member 4 (SMAD4) and cyclin dependent kinase inhibitor 2A (CDKN2A) is related to the development of pancreatic cancer (Pihlak et al. 2018). Obviously, mutations in tumor suppressor genes are linked to the progression of pancreatic cancer. Undoubtedly, an early diagnosis is beneficial for patients. Therefore, accurate knowledge of the molecular mechanisms involved in the tumorigenesis and proliferation of pancreatic cancer is vital.

Microarray technology and bioinformatic analysis have been extensively applied for screening the expression of genes, miRNAs, lncRNAs, and DNA methylation, helping to identifyDEGs and functional pathways relevant to the tumorigenesis and progression of pancreatic cancer. In this work, we aimed to explore the pathogenesis of pancreatic cancer by a computational bioinformatics analysis of gene expression. Three mRNA microarray datasets from the GEO were extracted and analyzed to identify DEGs between PDAC tissues and noncancerous tissues. Subsequently, the functions of these DEGs were evaluated using Gene Ontology (GO) and Kyoto Encyclopedia of Genes and Genomes (KEGG) pathway enrichment analyses. A protein-protein interaction (PPI) network was visualized using Cytoscape, and the Literature Mining Gene Networks module in GenCLiP 2.0 showed that cyclin-dependent kinase 1 (CDK1) has strong interactions with other hub genes. Enrichment analysis of GenCLiP 2.0 suggested that cell division may be involved in the pathogenesis of 
81 pancreatic cancer. In the GEPIA database, the mRNA levels of all hub genes were higher in PAAD (pancreatic

82

83

84

85

86 adenocarcinoma) tumor tissues than in normal tissues. Additionally, survival analysis indicated that abnormal spindle microtubule assembly (ASPM), CDK1, centromere protein F (CENPF), centrosomal protein 55 (CEP55), denticleless E3 ubiquitin protein ligase homolog (DTL), epithelial cell transforming 2 (ECT2), NIMA related kinase 2 (NEK2) and protein regulator of cytokinesis 1 (PRC1) may be associated with the tumorigenesis and development of pancreatic cancer. Overall, 210 DEGs and 13 hub genes that may be candidate biomarkers for pancreatic cancer were identified.

\section{Materials and Methods}

\section{Microarray datasets and data processing}

Three human pancreatic cancer mRNA expression datasets [GSE32676 (Li et al. 2018a), GSE15471 (Lu \& Li 2018) and GSE71989 (Li et al. 2018a)] were downloaded from GEO (http://www.ncbi.nlm.nih.gov/geo) (Edgar et al. 2002), a public functional genomic database containing high-throughput gene expression data, chips and microarrays. They all used the GPL570 [HG-U133_Plus_2] Affymetrix Human Genome U133 Plus 2.0 Array; human PDAC tumors and nonmalignant pancreas samples snap-frozen at the time of surgery were chosen. The GSE32676 dataset contains 25 PDAC tissue samples and 7 nonmalignant pancreas samples, GSE15471 contains 39 PDAC tissue samples and 39 nonmalignant pancreas samples, and GSE71989 contains 13 PDAC samples and 8 noncancerous samples.

\section{Identification of DEGs}

\section{DEGs between PDAC and nonmalignant samples were screened by GEO2R} (http://www.ncbi.nlm.nih.gov/geo/geo2r) (Shao et al. 2018), which is an online tool that can be used to compare two or more datasets in a GEO series to identify DEGs according to experimental conditions. Adjusted P-values (adj. P) and Benjamini and Hochberg false discovery rates were employed as criteria for statistically significant genes and to limit false positives. Probe sets with no corresponding gene symbols or genes with multiple gene probe sets were removed or averaged. Log FC (fold change) $>1$ or $<-1$ and adj. $\mathrm{P}<0.01$ was considered statistically significant. An online tool (http://bioinformatics. psb.ugent.be/webtools/Venn/) was applied to draw Venn diagrams of the DEGs. 


\section{KEGG and GO enrichment analyses of DEGs}

The Database for DAVID; (david.ncifcrf.gov/) online tool was used to obtain a synthetic set of functional annotation information for the DEGs (Huang et al. 2007; Le et al. 2018; Le et al. 2017; Meel et al. 2019). P<0.01 was considered statistically significant.

\section{PPI network construction and module analysis}

A PPI network of the DEGs was established using the STRING (http://string-db.org, version 10.0) database (Szklarczyk et al. 2015), and a combination of more than 0.4 was considered statistically significant for the PPI. Subsequently, we visualized the PPI network using Cytoscape, which is an open-source bioinformatics software platform (Shannon et al. 2003). Functional module analysis was then performed using MCODE, which is an app for Cytoscape that is used to cluster a given network to a densely connected area based on topology. The standard for selection was set as follows: MCODE scores $>5$, degree cut-off $=2$, node score cut-off $=0.2$, max depth $=100$ and $\mathrm{k}$-score $=2($ Li et al. 2017a).

\section{Hub gene selection and analysis}

Genes with a degree $\geq 10$ were chosen as hub genes, and pathways of the hub genes were visualized using ClueGO and CluePedia, which are two plugins of Cytoscape (Bindea et al. 2013). Additionally, GenCLiP 2.0 (http://ci.smu.edu.cn) (Wang et al. 2014), which facilitates functional annotation and molecular network construction of genes depending on the literature, was used to explore interactions of hub genes. The Gene Cluster with Literature Profiles modules were used to generate statistically overrepresented keywords to annotate genes based on the occurrence of free terms in the literature for a given gene. $\mathrm{P} \leq 1 \times 10^{6}$ and hits $\geq 6$ were considered statistically significant ( $\mathrm{Li}$ et al. 2017b). We selected keyword annotation to obtain a cluster analysis heatmap of 13 hub genes. The Literature Mining Gene Networks module was applied to explore the cocitation of hub genes. Furthermore, the expression levels of the 13 hub genes in pancreatic cancer tissues and normal samples were validated using the GEPIA platform, which is a free online database (http://gepia.cancer-pku.cn/) (Hauptman et al. 2019). Moreover, overall survival and disease-free survival analyses according to the expression of hub genes were conducted using Kaplan-Meier curve analysis in the cBioPortal (http://www.cbioportal.org) online platform (Gao et al. 2013). The relationship between expression level and 
133

134

135

136

137

138

139

140

141

142

tumor grade was analyzed using the online database Oncomine (http://www.oncomine.com) (Rhodes et al. 2004).

\section{Transcription factor analysis}

The Cytoscape plugin iRegulon (Janky et al. 2014) was employed to analyze transcription factors regulating the hub genes. The iRegulon plugin can identify regulons using motifs and track discovery in an existing network or in a set of coregulated genes. Transcription factor information is obtained from databases such as Transfac, Jaspar, Encode, Swissregulon and Homer, which use genome-wide ranking and recovery to detect enriched transcription factor motifs and optimal sets of their direct targets. The cutoff criteria were as follows: enrichment score threshold $=5.0$, ROC threshold for AUC calculation $=0.03$, rank threshold $=5000$, minimum identity between orthologous genes $=0.05, \mathrm{FDR}=0.001$ and the normalized enrichment score $(\mathrm{NES})>10$ ( $\mathrm{Li}$ et al. 2017b).

\section{Patients and tissue specimens}

The eight snap-frozen tumorous and adjacent noncancerous adjacent tissues of pancreatic cancer patients used to detect the CDK1 and CEP55 protein levels in this study were provided by the Affiliated Hospital of Nantong University. Our experimental protocols have been subjected to approval by the Institutional Review Board of Affiliated Hospital of Nantong University; all participating patients fully understood the protocols and subscribed informed consent. The cohort of patients included 6 female patients and 2 male patients who underwent surgical resection without chemotherapy or radiotherapy.

\section{Western blotting}

Western blotting was performed as previously described (Jiao et al. 2019). After blotting, the membranes were incubated at $4^{\circ} \mathrm{C}$ overnight with anti-CDK1 (ab18, diluted 1:1000, Abcam, MA, USA), anti-CEP55 (ab170414, diluted 1:1000, Abcam, MA), anti-PCNA (ab29, diluted 1:1000, Abcam, MA) and anti-GAPDH (\#5174; 1:5000; Cell Signaling Technology, MA, USA) antibodies. This was followed by incubation with antimouse IgG (\#D110087; 1:2500; Sangon Biotech, Shanghai, China) or anti-rabbit IgG (\#D110058; 1:2500; Sangon Biotech, Shanghai, China) secondary antibodies. Finally, an enhanced chemiluminescence (ECL) kit was used to visualize the bands, and the Molecular Imager ChemiDoc XRS System (Bio-Rad Laboratories, CA, USA) was used to analyze and quantify the bands. 


\section{Statistical analysis}

All data were analyzed using the GraphPad Prism 5.0 software. The results were presented as the mean \pm standard error of mean of at least three independent experiments. The comparison between multiple groups used single-factor analysis of variance, and the comparison of data between separate groups was performed using t test. $\mathrm{P}<0.05$ indicates statistical significance.

\section{Results}

Identification of DEGs in PDAC. In total, 775, 1793 and 3952 DEGs were identified when comparing PDAC tissue samples and normal tissue samples in the GSE32676, GSE15471 and GSE71989 datasets, respectively. Additionally, 210 (186 upregulated and 24 downregulated) genes were common to all three datasets (Fig. 1A).

PPI network construction. The PPI network of DEGs was constructed using the STRING database and included 133 genes (125 upregulated and 8 downregulated) with combined scores $>0.4$ (Fig. 1B).As the number of downregulated genes was too small for GO and KEGG enrichment analyses, we only performed this analysis for the upregulated genes.

\section{GO and KEGG pathway analyses of the PPI network. GO and KEGG pathway analyses} were conducted to explore the potential functions and pathways of the upregulated DEGs using DAVID. According to GO analysis, the upregulated DEGs were enriched in cell migration, cell-cell adhesion and cell adhesion biological process (BP) categories (Table 1). The upregulated DEGs were primarily enriched in the extracellular exosome and cytoplasm cell component (CC) categories (Table 1); DEGs were mainly enriched in the cadherin binding involved in cell-cell adhesion and protein homodimerization activity molecular function (MF) categories (Table 1). Additionally, KEGG pathway analysis indicated that the upregulated DEGs were primarily enriched in the ECM-receptor interaction and pathways in cancer (Table 2).

Hub gene selection and analysis. Thirteen genes were considered to be hub genes with a degree $\geq 10$ (Fig. 1C). Detailed information about these hub genes is presented in Table 3 . The pathways of the hub genes were visualized using ClueGO and CluePedia, which are two plugins of Cytoscape (Fig. 2). Subsequently, 
literature mining was performed in GenCLiP 2.0 to explore hub gene interactions. The cocitation network of these 13 hub genes in the published literature is displayed in Fig. 3A. The results showed that 5 genes, DNA topoisomerase II alpha (TOP2A), PRC1, ECT2, RRM2 and CEP55 interact with CDK1. TOP2A was the top gene because it has been mentioned in 23 published literature sources as interacting with CDK1. The detailed results of previous studies on these genes are shown in Table 4. Fig. 3B illustrates the significant results from the enrichment analysis of the 13 hub genes, with cell division being the most related biological function at $76.9 \%$ enrichment.

Transcription factor analysis of hub genes. Transcription factor analysis of the 13 hub genes was conducted using iRegulon, a Cytoscape plugin, and a normalized enrichment score (NES) $>10$ was considered to be significant. The transcriptional regulation network of these hub genes is shown in Fig. 3C. The transcription factors with NES $>10$ were NFYC $(\mathrm{NES}=15.131$, targets=11), TFDP3 (NES=11.922, targets=11), NFYA (NES=11.193, targets=9), and E2F4 (NES=10.426, targets=13).

\section{mRNA expression levels of the 13 hub genes in PAAD. To confirm the expression levels}

of the 13 identified hub genes, related published data were obtained from TCGA datasets and analyzed using the GEPIA platform. As expected, the results showed that all hub genes were more highly expressed in tumor tissues than in normal tissue samples (Fig. 4).

\section{Survival analyses of hub genes. Kaplan-Meier curve analysis was used to analyze correlations}

between overall survival and the hub genes. PDAC patients with alterations in anillin actin-binding protein (ANLN), ASPM, CDK1, CENPF, CEP55, DTL, ETC2, NEK2, TOP2A and PRC1 exhibited poor overall survival (Fig. 5). PDAC patients with ASPM, CDK1, CENPF, CEP55, DTL, ETC2, NEK2, PRC1, ribonucleotide reductase regulatory subunit M2 (RRM2), TOP2A, ZW10 interacting kinetochore protein (ZWINT), and ANLN alterations exhibited poor disease-free survival (Fig. 6). Among these genes, we selected CDK1 and CEP55 for further study with regard to overall survival and disease-free survival times.

\section{Oncomine analysis of CDK1 and CEP55 in cancer vs. normal tissue. Oncomine} analysis of cancer vs. normal tissue indicated that CDK1 and CEP55 were significantly overexpressed in pancreatic cancer in different datasets (Fig. 7A and B). In the Grutzmann Pancreas dataset, CDK1 and CEP55 
210

211

212

213

214

215

mRNA expression was higher in pancreatic cancer tissues than in normal pancreatic tissues (Fig. 7C and D). Additionally, higher mRNA levels of CDK1 and CEP55 were associated with tumor grade (Fig. 7E and F).

\section{The expression of CDK1 and CEP55 in clinical specimens. To validate the above} results, we detected CDK1 and CEP55 protein levels in clinical specimens. In the clinical specimens, CDK1 (Fig. 8A and C) and CEP55 (Fig. 8B and D) protein levels were significantly elevated in pancreatic cancer tissue samples compared with adjacent nontumor tissues.

\section{Discussion}

In recent years, microarray technology has been extensively applied to reveal genetic alterations in tumors. Therefore, microarray analysis is a tool for revealing biomarkers for the diagnosis, treatment, and prognosis of pancreatic cancer. In this study, our results demonstrated that the upregulated DEGs were obviously enriched in the BP categories cell migration and cell-cell adhesion. Many studies have shown that migration and invasion are basic characteristics of pancreatic cancer (Zhuo et al. 2018). Moreover, cell-cell adhesion might be involved in cell stretching and movement, which are the molecular bases of the important physiological and pathological processes of tumor invasion and metastasis (Nobes \& Hall 1999; Serrill et al. 2018). For the CC category, the upregulated DEGs were primarily enriched in extracellular exosomes, which are nanoscale membrane vesicles with diameters ranging from 40 to $100 \mathrm{~nm}$. A growing number of studies have shown that tumor-derived exosomes are associated with tumor development, metastasis, and drug resistance mechanisms (Jiao et al. 2018). Furthermore, in the MF category, upregulated DEGs were mainly enriched in cadherin binding involved in cellcell adhesion and protein homodimerization activity, which are associated with invasion and metastasis. According to our KEGG pathway enrichment results, the upregulated DEGs were primarily enriched in the ECM-receptor interaction. During the development of tumors, the ECM experiences a remodeling process that is similar to the process of embryonic development. The most important feature of this remodeling is the change in the molecular composition of the ECM, whereby the reconstituted ECM creates a loose microenvironment for the proliferation and differentiation of tumor cells, leading to high rates of proliferation, poor differentiation, invasion and metastasis (Jin \& Liu 2018).

$$
\text { In our study, we showed that TOP2A, PRC1, ECT2, RRM2 and CEP55 can interact with CDK1 and TOP2A }
$$

is the gene most closely related to CDK1. Previous studies have provided evidence about the expression of 
237 CDK1 and TOP2A in pancreatic cancer, but the correlation between them in pancreatic cancer has not been fully 238 elucidated (Kalimutho et al. 2018; Li et al. 2017a; Shi et al. 2015; Xu et al. 2016). Cyclin-dependent kinases 239 (CDKs) are important driving factors of the human cell cycle (Zhao et al. 2013). Topoisomerases, including 240 topoisomerase 1 (TOP1) and topoisomerase 2 (TOP2), are key ribozymes that mainly participate in cell growth 241 by breaking and reconnecting DNA strands to change DNA topology (Liu et al. 2018). We predict that the cyclin 242 A2-CDK1-TOP2A axis plays an important role in tumor development. Furthermore, CDK1 might phosphorylate 243 TOP2A to promote S phase transition and influence the progression of pancreatic cancer, but follow-up research 244 is needed to determine the specific mechanism of the interaction (Kalimutho et al. 2018). Our enrichment 245 analysis of 13 hub genes further verified the relationship between metastasis and the malignant progression of 246 pancreatic cancer.

247 The transcription factors NFYC, TFDP3, POLE3 and E2F4 are closely linked to hub genes in pancreatic 248 cancer. NFYC is a histone-fold domain-containing transcription factor engaged in chromatin remodeling, establishing permissive chromatin modifications at CCAAT motifs in promoters (Bieniossek et al. 2013). Deletion of NFYC halts cell cycle progression, predominantly by causing G2/M arrest, and concurrent gain of NFYC may serve to model an aberrant epigenome that promotes a proliferative and relatively undifferentiated state (Benatti et al. 2011). Most E2Fs are localized in the nucleus, but E2F4 shows cell cycle-specific localization and can be found in the nucleus of cycling cells in G0, early G1 and G2 phases (Lindeman et al. 1997). When present in the nucleus, E2F4 has functions necessary for the induction of mitosis; interestingly, E2F4 also appears to have a role in the cytoplasm during multiciliogenesis (van Amerongen et al. 2010). TFDP3 is expressed in most cancer tissues and potentially plays a role in cell differentiation and proliferation. TFDP3 is a novel negative regulator of E2F that can enhance both the DNA binding and transcriptional activity of E2F through the formation of heterodimers; it also potentially plays an important role in the process of tumor development independent of $\mathrm{pRb}$ (Ma et al. 2014). POLE3 is similar to the first subclass of core histones with respect to regulation, and POLE3 expression is upregulated at the onset of S phase. E2F4 is often associated with promoters in G0, is minimally bound to the POLE3 gene in starved cells and maximally in cells expressing POLE3 at high levels. These findings indicate that these transcription factors may regulate the progression of pancreatic cancer, which provides direction for our future research (Bolognese et al. 2006). 

levels of TOP2A (Jiao et al. 2019), CDK1 (Jing et al. 2019), RRM2 (Zhao et al. 2019), PRC1 (Mao et al. 2019), NEK2 (Deng et al. 2019), ZWINT (Obuse et al. 2004), DTL(Cui et al. 2019), MELK (Meel et al. 2019), CENPF (Chen et al. 2019), CEP55 (Hauptman et al. 2019), ANLN (Wang et al. 2019), ASPM (Hsu et al. 2019), and ECT2 (Daulat et al. 2019) in tumor tissues. Previous studies have provided abundant evidence about the function of the thirteen identified hub genes in cancer development. Mutations in ANLN, ASPM, CDK1, CENPF, CEP55, DTL, ETC2, NEK2, TOP2A and PRC1 affect overall survival and disease-free survival in pancreatic cancer, whereas mutations in RRM2 and ZWINT affect disease-free survival. Hence, we reasoned that ANLN, ASPM,

CDK1, CENPF, CEP55, DTL, ETC2, NEK2, TOP2A and PRC1 might be preferable prognostic factors that are positively related to pancreatic cancer. Additionally, Wenzong $\mathrm{Lu}$ et al analyzed the microarray datasets GSE32676, GSE15471, GSE71989 and GSE19650 to identify five upregulated hub genes, including MELK, MET, THBS1, TOP2A and SDC1 (Lu et al. 2019); Jun Li et al analyzed the microarray datasets GSE71989, GSE15471, GSE16515, GSE32676, GSE41368 and GSE28735 and found that five genes (BIRC5, CKS2, ITGA3, ITGA6 and RALA) were significantly associated with survival time in patients with pancreatic duct adenocarcinoma (Li et al. 2018a); Tao Zhu et al studied the five GEO datasets (GSE15471, GSE16515, GSE18670, GSE32676, GSE71989) and reported that GJB2 and ERO1LB dysregulation was associated with tumorigenesis in pancreatic adenocarcinoma (Zhu et al. 2017). Within addition to these hub genes, we supplied some new hub genes that may be associated with the tumorigenesis and development of pancreatic cancer.

Our results showed that among these hub genes, TOP2A is the most closely related gene to CDK1, TOP2A and CDK1, and it has been previously reported as a biomarker for pancreatic cancer (Kokkinakis et al. 2005); however, to date, little attention has been paid to CEP55 and its possible relationship with CDK1. Indeed, only one study reported that CEP55 can inhibit CDK1 phosphorylation and proteolysis mediated by the anaphasepromoting complex to induce anaphase I in oocytes (Zhou et al. 2019). Nonetheless, the relationship between CEP55 and CDK1 and the mechanism involved have not been studied in pancreatic cancer cells. It is well known the fact that it readily metastasizes to and invades adjacent organs. CEP55 belongs to the centrosomal family of 
291 expressed in multiple cancers, such as colon cancer, hepatocellular carcinoma and bladder cancer (Gao \& Wang 292 2015; Li et al. 2018b; Singh et al. 2015). Moreover, overexpression of CEP55 accelerates the cell cycle transition 293 in gastric cancer, and low expression of CEP55 inhibits cell growth in breast cancer and gastric cancer 294 (Kalimutho et al. 2018; Tao et al. 2014). These results demonstrate that CEP55 may serve an oncogenic role and 295 a potential target for tumor treatment. CEP55 can also promote aggressive behavior in pancreatic cancer cells 296 by activating the NF-KB pathway (Peng et al. 2017a). Hence, the underlying mechanism of the proinvasive and 297 prometastatic effects of CEP55 in pancreatic cancer needs to be further studied. Last, we preliminarily validated 298 the ChIP analyses by detecting CDK1 and CEP55 protein levels in eight snap-frozen tumorous and adjacent noncancerous adjacent tissues of pancreatic cancer patients. The results showed that CDK1 and CEP55 were significantly overexpressed in pancreatic tumorous tissues compared to normal tissues, which is consistent with the results in different datasets. However, our sample size was relatively small, so we could not verify the link between clinical samples and prognosis. Fortunately, Piao Junjie et al validated that high expression of CDK1 was correlated with the short survival of pancreatic cancer patients by analyzing 99 cases of surgically resected pancreatic cancer samples and 71 cases of normal pancreases (Piao et al. 2019). Tao Peng et al reported that CEP55 expression was an independent prognostic factor of patient outcome and that CEP55 protein expression levels in pancreatic cancer specimens were inversely correlated with survival time by analyzing 126 archived paraffin-embedded pancreatic cancer specimens with immunohistochemical staining using an antibody against human CEP55 (Peng et al. 2017b). The results were in accordance with the Kaplan-Meier curve analysis online.

\section{Conclusion}

In summary, by analyzing multiple datasets from the GEO database and validating the results with the TCGA and Oncomine databases, our present work identifies dominant genes, their interaction network and possible transcription factors involved during the progression and metastasis of pancreatic cancer. Some relationships between hub genes and transcription factors have never been reported to influence the progression of pancreatic cancer and may serve as potential targets for pancreatic cancer therapy. However, due to the low quantity of gene probes in our selected datasets, the number of discovered DEGs was strikingly limited. More 
317

318

319

320

321

322

323

324

325

326

327

328

329

330

331

332

333

334

335

336

337

338

339

340

341

342

343

344

345

346

347

348

349

350

understanding of pancreatic cancer development. In addition, we did not analyze the expression of hub genes, such as CDK1, in pancreatic patients with or without lymphatic metastasis or use ROC analysis to explain the prognosis and diagnostic value of the genes. We will collect these data in our future research to complete our analysis of the prognosis and diagnostic value of these genes.

\section{Data Availability}

The datasets generated and analyzed during the current study are available in the GEO repository. GSE32676: https://www.ncbi.nlm.nih.gov/geo/query/acc.cgi?acc=GSE32676 GSE15471: https://www.ncbi.nlm.nih.gov/geo/query/acc.cgi?acc=GSE15471 GSE71989: https://www.ncbi.nlm.nih.gov/geo/query/acc.cgi?acc=GSE71989

\section{References}

Becker A, Thakur BK, Weiss JM, Kim HS, Peinado H, and Lyden D. 2016. Extracellular Vesicles in Cancer: Cell-to-Cell Mediators of Metastasis. Cancer Cell 30:836-848. 10.1016/j.ccell.2016.10.009

Benatti P, Dolfini D, Vigano A, Ravo M, Weisz A, and Imbriano C. 2011. Specific inhibition of NF-Y subunits triggers different cell proliferation defects. Nucleic Acids Res 39:5356-5368. 10.1093/nar/gkr128

Bieniossek C, Papai G, Schaffitzel C, Garzoni F, Chaillet M, Scheer E, Papadopoulos P, Tora L, Schultz P, and Berger I. 2013. The architecture of human general transcription factor TFIID core complex. Nature 493:699-702. 10.1038/nature11791

Bindea G, Galon J, and Mlecnik B. 2013. CluePedia Cytoscape plugin: pathway insights using integrated experimental and in silico data. Bioinformatics 29:661-663. 10.1093/bioinformatics/btt019

Bolognese F, Forni C, Caretti G, Frontini M, Minuzzo M, and Mantovani R. 2006. The Pole3 bidirectional unit is regulated by MYC and E2Fs. Gene 366:109-116. 10.1016/j.gene.2005.07.046

Caldas C, Hahn SA, Hruban RH, Redston MS, Yeo CJ, and Kern SE. 1994. Detection of K-ras mutations in the stool of patients with pancreatic adenocarcinoma and pancreatic ductal hyperplasia. Cancer Res 54:3568-3573.

Caruso Bavisotto C, Cappello F, Macario AJL, Conway de Macario E, Logozzi M, Fais S, and Campanella C. 2017. Exosomal HSP60: a potentially useful biomarker for diagnosis, assessing prognosis, and monitoring response to treatment. Expert Rev Mol Diagn 17:815-822. $10.1080 / 14737159.2017 .1356230$

Chen EB, Qin X, Peng K, Li Q, Tang C, Wei YC, Yu S, Gan L, and Liu TS. 2019. HnRNPR-CCNB1/CENPF axis contributes to gastric cancer proliferation and metastasis. Aging (Albany NY) 11. 10.18632/aging.102254

Cui H, Wang Q, Lei Z, Feng M, Zhao Z, Wang Y, and Wei G. 2019. DTL promotes cancer progression by PDCD4 ubiquitin-dependent degradation. J Exp Clin Cancer Res 38:350. 10.1186/s13046-019-1358-x

Daulat AM, Finetti P, Revinski D, Silveira Wagner M, Camoin L, Audebert S, Birnbaum D, Kodjabachian L, 
351

352

353

354

355

356

357

358

359

360

361

362

363

364

365

366

367

368

369

370

371

372

373

374

375

376

377

378

379

380

381

382

383

384

385

386

387

388

389

390

391

Borg JP, and Bertucci F. 2019. ECT2 associated to PRICKLE1 are poor-prognosis markers in triplenegative breast cancer. Br J Cancer 120:931-940. 10.1038/s41416-019-0448-Z

Deng L, Sun J, Chen X, Liu L, and Wu D. 2019. Nek2 augments sorafenib resistance by regulating the ubiquitination and localization of beta-catenin in hepatocellular carcinoma. J Exp Clin Cancer Res 38:316. 10.1186/s13046-019-1311-z

Edgar R, Domrachev M, and Lash AE. 2002. Gene Expression Omnibus: NCBI gene expression and hybridization array data repository. Nucleic Acids Res 30:207-210.

Gao J, Aksoy BA, Dogrusoz U, Dresdner G, Gross B, Sumer SO, Sun Y, Jacobsen A, Sinha R, Larsson E, Cerami E, Sander C, and Schultz N. 2013. Integrative analysis of complex cancer genomics and clinical profiles using the cBioPortal. Sci Signal 6:pl1. 10.1126/scisignal.2004088

Gao XY, and Wang XL. 2015. An adoptive T cell immunotherapy targeting cancer stem cells in a colon cancer model. J buon 20:1456-1463.

Hauptman N, Jevsinek Skok D, Spasovska E, Bostjancic E, and Glavac D. 2019. Genes CEP55, FOXD3, FOXF2, GNAO1, GRIA4, and KCNA5 as potential diagnostic biomarkers in colorectal cancer. BMC Med Genomics 12:54. 10.1186/s12920-019-0501-z

Hsu CC, Liao WY, Chan TS, Chen WY, Lee CT, Shan YS, Huang PJ, Hou YC, Li CR, and Tsai KK. 2019. The differential distributions of ASPM isoforms and their roles in Wnt signaling, cell cycle progression, and pancreatic cancer prognosis. J Pathol. 10.1002/path.5341

Huang DW, Sherman BT, Tan Q, Collins JR, Alvord WG, Roayaei J, Stephens R, Baseler MW, Lane HC, and Lempicki RA. 2007. The DAVID Gene Functional Classification Tool: a novel biological modulecentric algorithm to functionally analyze large gene lists. Genome Biol 8:R183. 10.1186/gb-2007-8-9r183

Janky R, Verfaillie A, Imrichova H, Van de Sande B, Standaert L, Christiaens V, Hulselmans G, Herten K, Naval Sanchez M, Potier D, Svetlichnyy D, Kalender Atak Z, Fiers M, Marine JC, and Aerts S. 2014. iRegulon: from a gene list to a gene regulatory network using large motif and track collections. PLoS Comput Biol 10:e1003731. 10.1371/journal.pcbi.1003731

Jiao YJ, Jin DD, Jiang F, Liu JX, Qu LS, Ni WK, Liu ZX, Lu CH, Ni RZ, Zhu J, and Xiao MB. 2018. Characterization and proteomic profiling of pancreatic cancer-derived serum exosomes. $10.1002 / \mathrm{jcb} .27465$

Jiao YJ, Jin DD, Jiang F, Liu JX, Qu LS, Ni WK, Liu ZX, Lu CH, Ni RZ, Zhu J, and Xiao MB. 2019. Characterization and proteomic profiling of pancreatic cancer-derived serum exosomes. J Cell Biochem 120:988-999. 10.1002/jcb.27465

Jin Q, and Liu G. 2018. Decellularized breast matrix as bioactive microenvironment for in vitro threedimensional cancer culture. 10.1002/jcp.26782

Jing X, Wang XJ, Zhang T, Zhu W, Fang Y, Wu H, Liu X, Ma D, Ji X, Jiang Y, Liu K, Chen X, Shi Y, Zhang Y, Shi M, Qiu W, and Zhao R. 2019. Cell-Cycle-Dependent Phosphorylation of PRPS1 Fuels Nucleotide Synthesis and Promotes Tumorigenesis. Cancer Res 79:4650-4664. 10.1158/00085472.can-18-2486

Kalimutho M, Sinha D, Jeffery J, Nones K, Srihari S, Fernando WC, Duijf PH, Vennin C, Raninga P, Nanayakkara D, Mittal D, Saunus JM, Lakhani SR, Lopez JA, Spring KJ, Timpson P, Gabrielli B, Waddell N, and Khanna KK. 2018. CEP55 is a determinant of cell fate during perturbed mitosis in 
392

393

394

395

396

397

398

399

400

401

402

403

404

405

406

407

408

409

410

411

412

413

414

415

416

417

418

419

420

421

422

423

424

425

426

427

428

429

430

431

432

breast cancer. EMBO Mol Med 10. 10.15252/emmm.201708566

Kokkinakis DM, Liu X, and Neuner RD. 2005. Modulation of cell cycle and gene expression in pancreatic tumor cell lines by methionine deprivation (methionine stress): implications to the therapy of pancreatic adenocarcinoma. Mol Cancer Ther 4:1338-1348. 10.1158/1535-7163.mct-05-0141

Le NQ, Ho QT, and Ou YY. 2018. Classifying the molecular functions of Rab GTPases in membrane trafficking using deep convolutional neural networks. Anal Biochem 555:33-41. 10.1016/j.ab.2018.06.011

Le NQ, Nguyen TT, and Ou YY. 2017. Identifying the molecular functions of electron transport proteins using radial basis function networks and biochemical properties. J Mol Graph Model 73:166-178. 10.1016/j.jmgm.2017.01.003

Li J, Tan W, Peng L, Zhang J, Huang X, Cui Q, Zheng J, Tan W, Wu C, and Lin D. 2018a. Integrative analysis of gene expression profiles reveals specific signaling pathways associated with pancreatic duct adenocarcinoma. Cancer Commun (Lond) 38:13. 10.1186/s40880-018-0289-9

Li L, Lei Q, Zhang S, Kong L, and Qin B. 2017a. Screening and identification of key biomarkers in hepatocellular carcinoma: Evidence from bioinformatic analysis. Oncol Rep 38:2607-2618. 10.3892/or.2017.5946

Li L, Wang G, Li N, Yu H, Si J, and Wang J. 2017b. Identification of key genes and pathways associated with obesity in children. Exp Ther Med 14:1065-1073. 10.3892/etm.2017.4597

Li M, Gao J, Li D, and Yin Y. 2018b. CEP55 Promotes Cell Motility via JAK2(-)STAT3(-)MMPs Cascade in Hepatocellular Carcinoma. Cells 7. 10.3390/cells7080099

Lindeman GJ, Gaubatz S, Livingston DM, and Ginsberg D. 1997. The subcellular localization of E2F-4 is cellcycle dependent. Proc Natl Acad Sci U S A 94:5095-5100. 10.1073/pnas.94.10.5095

Liu LM, Xiong DD, Lin P, Yang H, Dang YW, and Chen G. 2018. DNA topoisomerase 1 and 2A function as oncogenes in liver cancer and may be direct targets of nitidine chloride. Int J Oncol 53:1897-1912. 10.3892/ijo.2018.4531

Lu W, Li N, and Liao F. 2019. Identification of Key Genes and Pathways in Pancreatic Cancer Gene Expression Profile by Integrative Analysis. Genes (Basel) 10. 10.3390/genes 10080612

$\mathrm{Lu} \mathrm{Y}$, and Li C. 2018. Identification of hub genes and analysis of prognostic values in pancreatic ductal adenocarcinoma by integrated bioinformatics methods. 10.1007/s11033-018-4325-2

Ma Y, Xin Y, Li R, Wang Z, Yue Q, Xiao F, and Hao X. 2014. TFDP3 was expressed in coordination with E2F1 to inhibit E2F1-mediated apoptosis in prostate cancer. Gene 537:253-259. 10.1016/j.gene.2013.12.051

Mao J, Tian Y, Wang C, Jiang K, Li R, Yao Y, Zhang R, Sun D, Liang R, Gao Z, Wang Q, and Wang L. 2019. CBX2 Regulates Proliferation and Apoptosis via the Phosphorylation of YAP in Hepatocellular Carcinoma. J Cancer 10:2706-2719. 10.7150/jca.31845

Meel MH, Guillen Navarro M, de Gooijer MC, Metselaar DS, Waranecki P, Breur M, Lagerweij T, Wedekind LE, Koster J, van de Wetering MD, Schouten-van Meeteren N, Aronica E, van Tellingen O, Bugiani M, Phoenix TN, Kaspers GJL, and Hulleman E. 2019. MEK/MELK inhibition and blood-brain barrierdeficiencies in atypical teratoid/rhabdoid tumors. Neuro Oncol. 10.1093/neuonc/noz151

Nobes CD, and Hall A. 1999. Rho GTPases control polarity, protrusion, and adhesion during cell movement. J Cell Biol 144:1235-1244.

Obuse C, Iwasaki O, Kiyomitsu T, Goshima G, Toyoda Y, and Yanagida M. 2004. A conserved Mis12 centromere complex is linked to heterochromatic HP1 and outer kinetochore protein Zwint-1. Nat Cell 
433

434

435

436

437

438

439

440

441

442

443

444

445

446

447

448

449

450

451

452

453

454

455

456

457

458

459

460

461

462

463

464

465

466

467

468

469

470

471

472

473

Biol 6:1135-1141. 10.1038/ncb1187

Peng T, Zhou W, Guo F, Wu HS, Wang CY, Wang L, and Yang ZY. 2017a. Centrosomal protein 55 activates NF-kappaB signalling and promotes pancreatic cancer cells aggressiveness. Sci Rep 7:5925. 10.1038/s41598-017-06132-z

Peng T, Zhou W, Guo F, Wu HS, Wang CY, Wang L, and Yang ZY. 2017b. Centrosomal protein 55 activates $\mathrm{NF}-\kappa \mathrm{B}$ signalling and promotes pancreatic cancer cells aggressiveness. Sci Rep 7:5925. 10.1038/s41598-017-06132-z

Piao J, Zhu L, Sun J, Li N, Dong B, Yang Y, and Chen L. 2019. High expression of CDK1 and BUB1 predicts poor prognosis of pancreatic ductal adenocarcinoma. Gene 701:15-22. 10.1016/j.gene.2019.02.081

Pihlak R, Weaver JMJ, Valle JW, and McNamara MG. 2018. Advances in Molecular Profiling and Categorisation of Pancreatic Adenocarcinoma and the Implications for Therapy. Cancers (Basel) 10. $10.3390 /$ cancers 10010017

Rhodes DR, Yu J, Shanker K, Deshpande N, Varambally R, Ghosh D, Barrette T, Pandey A, and Chinnaiyan AM. 2004. ONCOMINE: a cancer microarray database and integrated data-mining platform. Neoplasia 6:1-6.

Serrill JD, Sander M, and Shih HP. 2018. Pancreatic Exocrine Tissue Architecture and Integrity are Maintained by E-cadherin During Postnatal Development. 8:13451. 10.1038/s41598-018-31603-2

Shannon P, Markiel A, Ozier O, Baliga NS, Wang JT, Ramage D, Amin N, Schwikowski B, and Ideker T. 2003. Cytoscape: a software environment for integrated models of biomolecular interaction networks. Genome Res 13:2498-2504. 10.1101/gr.1239303

Shao N, Yuan K, Zhang Y, Yun Cheang T, Li J, and Lin Y. 2018. Identification of key candidate genes, pathways and related prognostic values in ER-negative/HER2-negative breast cancer by bioinformatics analysis. J buon 23:891-901.

Shi Z, Chiang CI, Labhart P, Zhao Y, Yang J, Mistretta TA, Henning SJ, Maity SN, and Mori-Akiyama Y. 2015. Context-specific role of SOX9 in NF-Y mediated gene regulation in colorectal cancer cells. Nucleic Acids Res 43:6257-6269. 10.1093/nar/gkv568

Siegel RL, Miller KD, and Jemal A. 2018. Cancer statistics, 2018. 68:7-30. 10.3322/caac.21442

Singh PK, Srivastava AK, Rath SK, Dalela D, Goel MM, and Bhatt ML. 2015. Expression and clinical significance of Centrosomal protein 55 (CEP55) in human urinary bladder transitional cell carcinoma. Immunobiology 220:103-108. 10.1016/j.imbio.2014.08.014

Szklarczyk D, Franceschini A, Wyder S, Forslund K, Heller D, Huerta-Cepas J, Simonovic M, Roth A, Santos A, Tsafou KP, Kuhn M, Bork P, Jensen LJ, and von Mering C. 2015. STRING v10: protein-protein interaction networks, integrated over the tree of life. Nucleic Acids Res 43:D447-452. 10.1093/nar/gku1003

Tao J, Zhi X, Tian Y, Li Z, Zhu Y, Wang W, Xie K, Tang J, Zhang X, Wang L, and Xu Z. 2014. CEP55 contributes to human gastric carcinoma by regulating cell proliferation. Tumour Biol 35:4389-4399. 10.1007/s13277-013-1578-1

van Amerongen MJ, Diehl F, Novoyatleva T, Patra C, and Engel FB. 2010. E2F4 is required for cardiomyocyte proliferation. Cardiovasc Res 86:92-102. 10.1093/cvr/cvp383

Wang A, Dai H, Gong Y, Zhang C, Shu J, Luo Y, Jiang Y, Liu W, and Bie P. 2019. ANLN-induced EZH2 upregulation promotes pancreatic cancer progression by mediating miR-218-5p/LASP1 signaling axis. 
J Exp Clin Cancer Res 38:347. 10.1186/s13046-019-1340-7

Wang JH, Zhao LF, Lin P, Su XR, Chen SJ, Huang LQ, Wang HF, Zhang H, Hu ZF, Yao KT, and Huang ZX. 2014. GenCLiP 2.0: a web server for functional clustering of genes and construction of molecular networks based on free terms. Bioinformatics 30:2534-2536. 10.1093/bioinformatics/btu241

Xu X, Zhou Y, Miao R, Chen W, Qu K, Pang Q, and Liu C. 2016. Transcriptional modules related to hepatocellular carcinoma survival: coexpression network analysis. Front Med 10:183-190. 10.1007/s11684-016-0440-4

Zhao H, Zheng GH, Li GC, Xin L, Wang YS, Chen Y, and Zheng XM. 2019. Long noncoding RNA LINC00958 regulates cell sensitivity to radiotherapy through RRM2 by binding to microRNA-5095 in cervical cancer. J Cell Physiol 234:23349-23359. 10.1002/jcp.28902

Zhao J, Han SX, Ma JL, Ying X, Liu P, Li J, Wang L, Zhang Y, Ma J, Zhang L, and Zhu Q. 2013. The role of CDK1 in apoptin-induced apoptosis in hepatocellular carcinoma cells. Oncol Rep 30:253-259. 10.3892/or.2013.2426

Zhou C, Hancock JL, Khanna KK, and Homer HA. 2019. First meiotic anaphase requires Cep55-dependent inhibitory Cdk1 phosphorylation. J Cell Sci. 10.1242/jcs.233379

Zhu T, Gao YF, Chen YX, Wang ZB, Yin JY, Mao XY, Li X, Zhang W, Zhou HH, and Liu ZQ. 2017. Genomescale analysis identifies GJB2 and ERO1LB as prognosis markers in patients with pancreatic cancer. Oncotarget 8:21281-21289. 10.18632/oncotarget.15068

Zhuo M, Yuan C, Han T, Cui J, Jiao F, and Wang L. 2018. A novel feedback loop between high MALAT-1 and low miR-200c-3p promotes cell migration and invasion in pancreatic ductal adenocarcinoma and is predictive of poor prognosis. BMC Cancer 18:1032. 10.1186/s12885-018-4954-9

\section{Figures}

Figure 1. Venn diagram, PPI network and the most significant modules of DEG. (A) Venn diagram analyzing the DEGs of the GSE32676, GSE15471 and GSE71989 datasets; an overlap of 210 genes was identified. (B) The PPI network of DEGs was constructed by Cytoscape. Upregulated genes are marked in red, and the depth of color represents the degree of gene interaction with other genes; downregulated genes are marked in green. (C) Interaction network of 13 hub genes.

Figure 2. GO and KEGG pathway analysis of hub genes using ClueGO and CluePedia.

Figure 3. Interactions, enrichment and transcription factor analysis of 13 hub genes. (A). The cocitation network of 13 hub genes; the number on the line shows the number of studies cocited. (B). Heatmap of enrichment analysis of 13 hub genes; the depth of green color represents the degree of enrichment. (C) Transcription factor analysis of hub genes; the red nodes represent hub genes, and the purple nodes represent transcription factors. 
508

509

510

511

512

513

514

515

516

517

518

519

520

521

522

523

524

525

526

527

528

529

530

531

532

9

\section{0}

31

Figure 4. The mRNA expression levels of 13 hub genes in PAAD. Published online data of gene mRNA expression levels were analyzed using the GEPIA platform. mRNA levels of these 13 hub genes, including TOP2A, CDK1, RRM2, PRC1, NEK2, ZWINT, DTL, MELK, CENPF, CEP55, ANLN, ASPM, and ECT2, were all higher in PAAD tissues than in normal tissues. ${ }^{*} \mathrm{P}<0.05$.

Figure 5. Overall survival analyses of hub genes performed using the cBioPortal online platform. A log rank test $\mathrm{P}<0.05$ was considered statistically significant.

Figure 6. Disease-free survival analyses of hub genes performed using the cBioPortal online platform. A $\log$ rank test $\mathrm{P}<0.05$ was considered statistically significant.

Figure 7. Oncomine analysis of (A) CDK1 and (B) CEP55 in cancer vs. normal tissue. Heat maps of CDK1 and CEP55 gene expression in clinical pancreatic cancer samples vs. normal tissues. 1. Pancreatic Ductal Adenocarcinoma Epithelia vs. Normal Grutzmann Pancreas, Neoplasia, 2004. 2. Pancreatic Carcinoma vs. Normal Pei Pancreas, Cancer Cell, 2009. 3. Pancreatic Carcinoma vs. Normal Segara Pancreas, Clin Cancer Res, 2005. (C) CDK1 mRNA expression and (D) CEP55 mRNA expression in pancreatic cancer compared with that in normal pancreatic tissues in the Grutzmann Pancreas dataset. Association between the expression of (E) CDK1 and (F) CEP55 and tumor grade in the Grutzmann Pancreas dataset. $\mathrm{P}<0.05$ was considered statistically significant.

Figure 8. The expression of CDK1 and CEP55 in clinical specimens. (A, B) Western blotting detection of CDK1 and CEP55 protein in eight snap-frozen tumorous and tissues of pancreatic cancer patients. (C, D) The bar chart reveled that the expression ratio of CDK1 and CEP55 to GAPDH by densitometry. Data are represented as the mean $\pm \operatorname{SEM}(* \mathrm{P}<0.05$, tumor tissues compared with the non-cancerous adjacent tissues).

(1)


Table $\mathbf{1}$ (on next page)

GO analysis of upregulated DEGs in Pancreatic ductal adenocarcinoma 
1 Table 1. GO analysis of upregulated DEGs in Pancreatic ductal adenocarcinoma

\begin{tabular}{|c|c|c|c|}
\hline GO ID & Description & Gene count & P-value \\
\hline \multicolumn{4}{|l|}{ GO-BP Terms } \\
\hline GO:0016477 & cell migration & 13 & $1.83 \mathrm{E}-07$ \\
\hline GO:0098609 & cell-cell adhesion & 13 & $2.08 \mathrm{E}-05$ \\
\hline GO:0007160 & cell-matrix adhesion & 8 & $3.55 \mathrm{E}-05$ \\
\hline GO:0022617 & extracellular matrix disassembly & 7 & $1.20 \mathrm{E}-04$ \\
\hline GO:0048333 & mesodermal cell differentiation & 4 & $1.58 \mathrm{E}-04$ \\
\hline GO:0031581 & hemidesmosome assembly & 4 & $2.10 \mathrm{E}-04$ \\
\hline GO:0090004 & $\begin{array}{l}\text { positive regulation of establishment of } \\
\text { protein localization to plasma membrane }\end{array}$ & 5 & $2.26 \mathrm{E}-04$ \\
\hline GO:0007155 & cell adhesion & 15 & $2.45 \mathrm{E}-04$ \\
\hline GO:0007229 & integrin-mediated signaling pathway & 7 & $5.08 \mathrm{E}-04$ \\
\hline \multicolumn{4}{|l|}{ GO-CC Terms } \\
\hline GO:0070062 & extracellular exosome & 63 & $1.50 \mathrm{E}-10$ \\
\hline GO:0005925 & focal adhesion & 16 & 7.65E-06 \\
\hline GO:0005913 & cell-cell adherens junction & 14 & $1.91 \mathrm{E}-05$ \\
\hline GO:0005615 & extracellular space & 28 & $3.05 \mathrm{E}-04$ \\
\hline GO:0005886 & plasma membrane & 61 & $5.23 \mathrm{E}-04$ \\
\hline GO:0005737 & cytoplasm & 72 & $9.52 \mathrm{E}-04$ \\
\hline GO:0009986 & cell surface & 15 & $9.53 \mathrm{E}-04$ \\
\hline \multicolumn{4}{|c|}{ GO-MF Terms } \\
\hline GO:0098641 & $\begin{array}{l}\text { cadherin binding involved in cell-cell } \\
\text { adhesion }\end{array}$ & 13 & $3.05 \mathrm{E}-05$ \\
\hline GO:0042803 & protein homodimerization activity & 18 & $8.70 \mathrm{E}-04$ \\
\hline GO:0005509 & calcium ion binding & 16 & 0.004808 \\
\hline GO:0005515 & protein binding & 102 & 0.013495 \\
\hline
\end{tabular}

$\mathrm{GO}$, gene ontology; $\mathrm{BP}$, biological process; $\mathrm{CC}$, cellular component; $\mathrm{MF}$, molecular function.

2 


\section{Table 2 (on next page)}

KEGG pathway enrichment analysis of upregulated DEGs in Pancreatic ductal adenocarcinoma 
1 Table 2. KEGG pathway enrichment analysis of upregulated DEGs in Pancreatic ductal adenocarcinoma

\begin{tabular}{llcc}
\hline ID & Description & Gene count & P-value \\
\hline hsa04512 & ECM-receptor interaction & 9 & $1.82 \mathrm{E}-06$ \\
hsa05200 & Pathways in cancer & 12 & 0.001424 \\
hsa05205 & Proteoglycans in cancer & 8 & 0.003332 \\
hsa04510 & Focal adhesion & 8 & 0.003924 \\
hsa04115 & p53 signaling pathway & 5 & 0.004128 \\
hsa04151 & PI3K-Akt signaling pathway & 9 & 0.019238 \\
\hline
\end{tabular}

KEGG, Kyoto Encyclopedia of Genes and Genomes.

2 
Table 3 (on next page)

Description of 13 hub genes of Pancreatic ductal adenocarcinoma 
1 Table 3. Description of 13 hub genes of Pancreatic ductal adenocarcinoma

\begin{tabular}{|c|c|c|c|}
\hline NO. & $\begin{array}{l}\text { Gene } \\
\text { symbol }\end{array}$ & Full name & Function \\
\hline 1 & TOP2A & $\begin{array}{l}\text { DNA topoisomerase } \\
\text { II alpha }\end{array}$ & $\begin{array}{l}\text { Controlling and altering the topologic states of } \\
\text { DNA during transcription. Involving in } \\
\text { chromosome condensation, chromatid } \\
\text { separation, and the relief of torsional stress. }\end{array}$ \\
\hline 2 & CDK1 & $\begin{array}{l}\text { cyclin dependent } \\
\text { kinase } 1\end{array}$ & $\begin{array}{l}\text { Essential for } \mathrm{G} 1 / \mathrm{S} \text { and } \mathrm{G} 2 / \mathrm{M} \text { phase transitions } \\
\text { of eukaryotic cell cycle and mitotic cyclins. }\end{array}$ \\
\hline 3 & RRM2 & $\begin{array}{l}\text { ribonucleotide } \\
\text { reductase regulatory } \\
\text { subunit } \mathrm{M} 2\end{array}$ & $\begin{array}{l}\text { Catalyzing the formation of } \\
\text { deoxyribonucleotides from ribonucleotides. }\end{array}$ \\
\hline 4 & PRC1 & $\begin{array}{l}\text { protein regulator of } \\
\text { cytokinesis } 1\end{array}$ & $\begin{array}{l}\text { A substrate of several CDKs and necessary for } \\
\text { polarizing parallel microtubules and } \\
\text { concentrating the factors responsible for } \\
\text { contractile ring assembly. }\end{array}$ \\
\hline 5 & NEK2 & $\begin{array}{l}\text { NIMA related kinase } \\
2\end{array}$ & $\begin{array}{l}\text { A serine/threonine-protein kinase that is } \\
\text { involved in mitotic regulation. }\end{array}$ \\
\hline 6 & ZWINT & $\begin{array}{l}\text { ZW10 interacting } \\
\text { kinetochore protein }\end{array}$ & Clearly involved in kinetochore function. \\
\hline 7 & DTL & $\begin{array}{l}\text { denticleless E3 } \\
\text { ubiquitin protein } \\
\text { ligase homolog }\end{array}$ & Cellular response to DNA damage stimulus. \\
\hline 8 & MELK & $\begin{array}{l}\text { maternal embryonic } \\
\text { leucine zipper kinase }\end{array}$ & $\begin{array}{l}\mathrm{G} 2 / \mathrm{M} \text { transition of mitotic cell cycle apoptotic } \\
\text { process. }\end{array}$ \\
\hline 9 & CENPF & centromere protein $\mathrm{F}$ & $\begin{array}{l}\text { Associating with the centromere-kinetochore } \\
\text { complex and playing a role in chromosome } \\
\text { segregation during mitotis. }\end{array}$ \\
\hline 10 & CEP55 & $\begin{array}{l}\text { centrosomal protein } \\
55\end{array}$ & $\begin{array}{l}\text { Cranial skeletal system development and } \\
\text { establishment of protein localization. }\end{array}$ \\
\hline 11 & ANLN & $\begin{array}{l}\text { anillin actin binding } \\
\text { protein }\end{array}$ & $\begin{array}{l}\text { An actin-binding protein that plays a role in } \\
\text { cell growth and migration, and in cytokinesis. }\end{array}$ \\
\hline 12 & ASPM & $\begin{array}{l}\text { abnormal spindle } \\
\text { microtubule } \\
\text { assembly }\end{array}$ & $\begin{array}{l}\text { Essential for normal mitotic spindle function } \\
\text { in embryonic neuroblasts and regulating } \\
\text { neurogenesis. }\end{array}$ \\
\hline 13 & ECT2 & $\begin{array}{l}\text { epithelial cell } \\
\text { transforming } 2\end{array}$ & $\begin{array}{l}\text { A guanine nucleotide exchange factor and } \\
\text { transforming protein that is related to Rho- } \\
\text { specific exchange factors and yeast cell cycle } \\
\text { regulators. }\end{array}$ \\
\hline
\end{tabular}




\section{Table 4(on next page)}

Hub genes identified by the present study using Genclip 2.0 
1 Table 4. Hub genes identified by the present study using Genclip 2.0

\begin{tabular}{cccc}
\hline Gene & Co-genes & Co-cite & Total \\
\hline CDK1 & 5 & 53 & 6822 \\
TOP2A & 1 & 23 & 6370 \\
RRM2 & 1 & 7 & 395 \\
CEP55 & 1 & 1 & 58 \\
PRC1 & 1 & 15 & 334 \\
ECT2 & 1 & 11 & 171 \\
\hline
\end{tabular}

2 
Figure 1

Venn diagram, PPI network and the most significant modules of DEG.

(A) Venn diagram analyzing the DEGs of GSE32676, GSE15471 and GSE71989 datasets, and an overlap of 210 genes was identified. (B) The PPI network of DEGs was constructed by Cytoscape. Upregulated genes are marked in red, and the depth of color represents the gene interaction degree with other genes; downregulated genes are marked in green. (C) Interaction network of 13 hub genes.

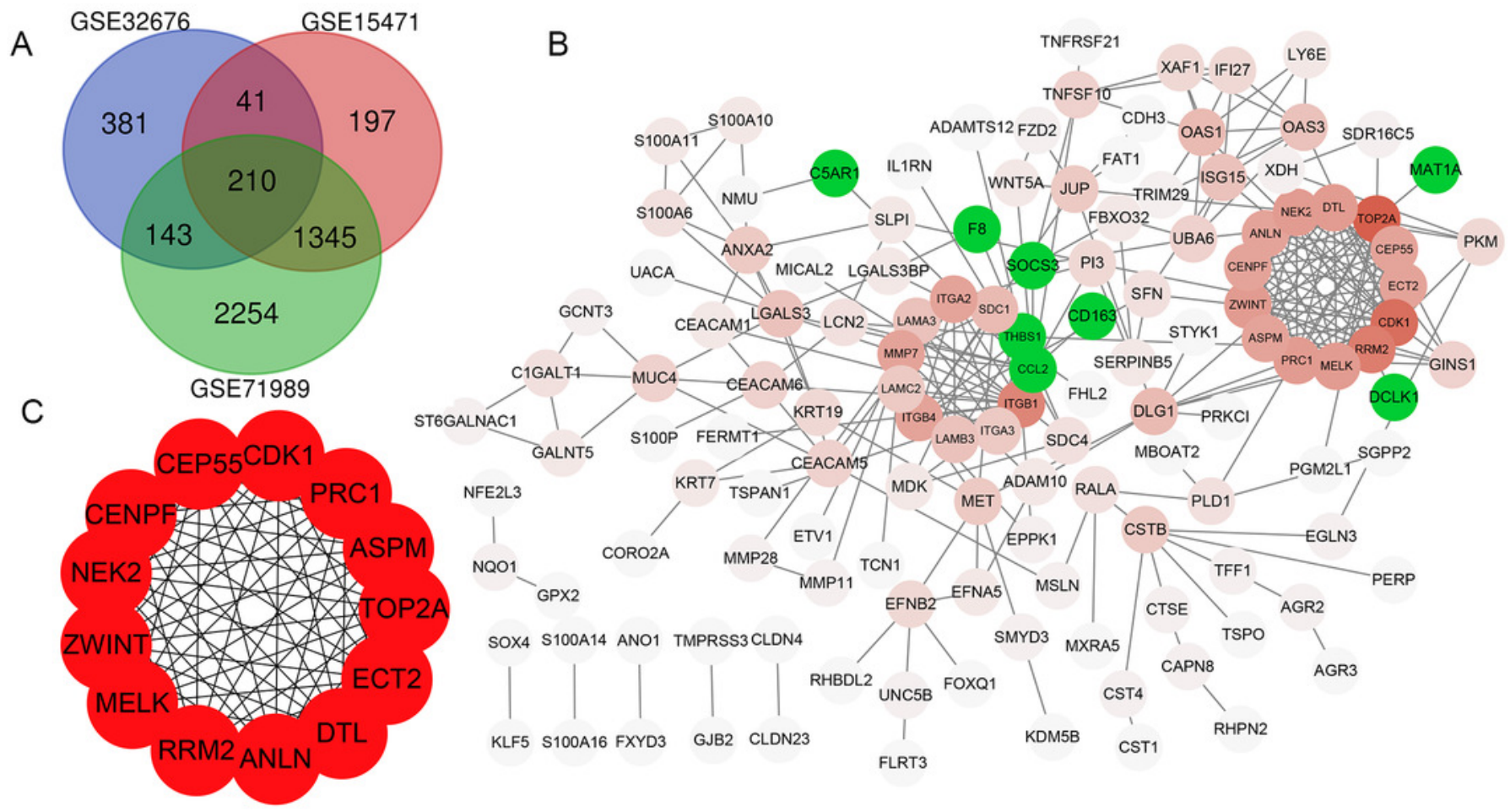


Figure 2

\section{GO and KEGG pathway analysis of hub genes using ClueGO and CluePedia.}






\section{Figure 3}

Interactions, enrichment and transcription factor analysis of 13 hubgenes.

(A). The cocitation network of 13 hub genes; the number on the line shows the number of studies cocited. (B). Heatmap of enrichment analysis of 13 hub genes; the depth of green color represents the degree of enrichment. (C) Transcription factor analysis of hub genes; the red nodes represent hub genes, and the purple nodes represent transcription factors.
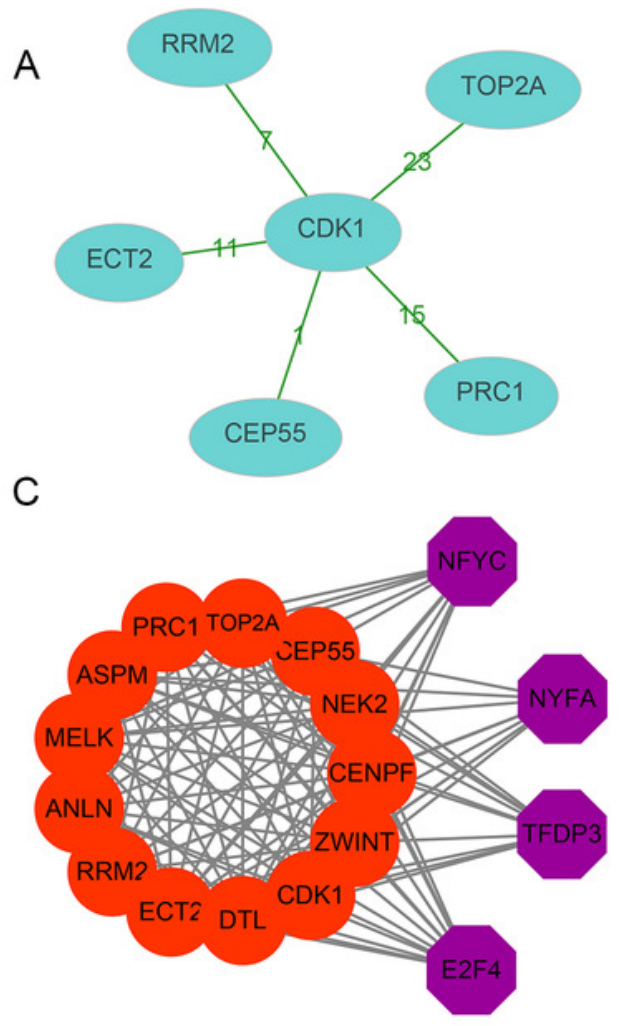

B

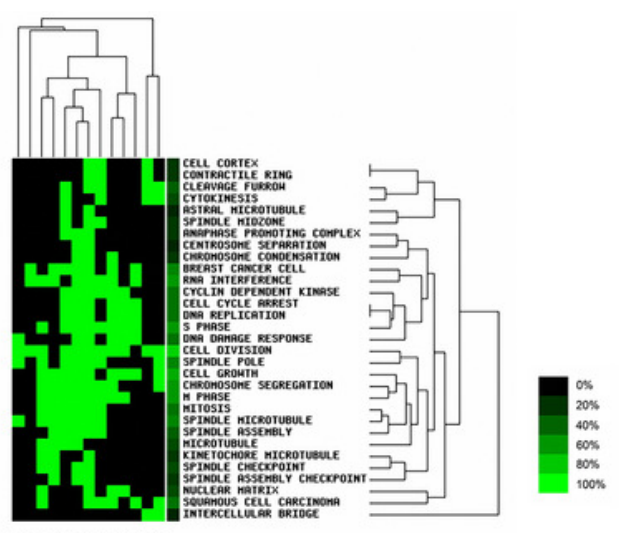




Figure 4

The mRNA expression levels of 13 hubgenes in PAAD.

The mRNA expression levels of $\mathbf{1 3}$ hubgenes in PAAD. The published online data of gene mRNA expression level were analyzed by GEPIA platform. These 13 hubgenes were all higher than in PAAD tissues, compared with those in normal tissues, including TOP2A, CDK1, RRM2, PRC1, NEK2, ZWINT, DTL, MELK, CENPF, CEP55, ANLN, ASPM, ECT2. *P<0.05.
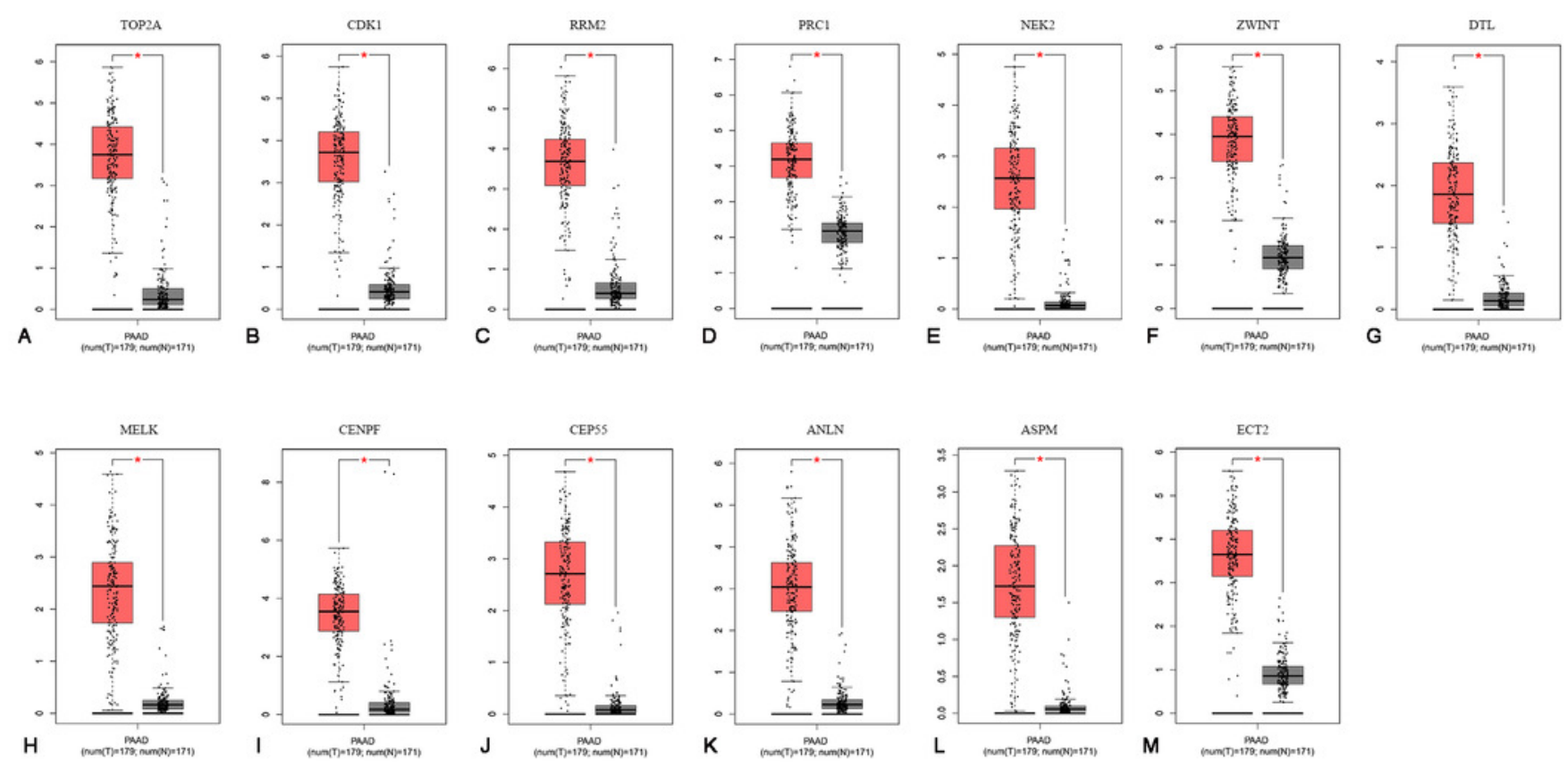
Figure 5

A log rank test $P<0.05$ was considered statistically significant.

Log rank Test $\mathrm{P}<0.05$ was considered statistically significant.

- Cases with Alteration(s) in Query Gene(s)

- Cases without Alteration(s) in Query Gene(s)
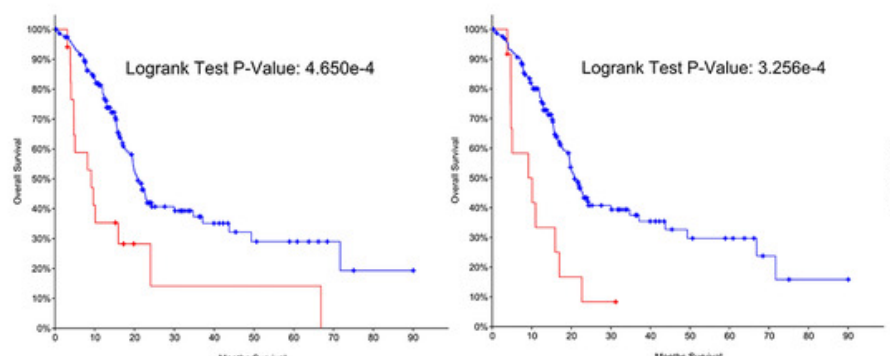

A

B
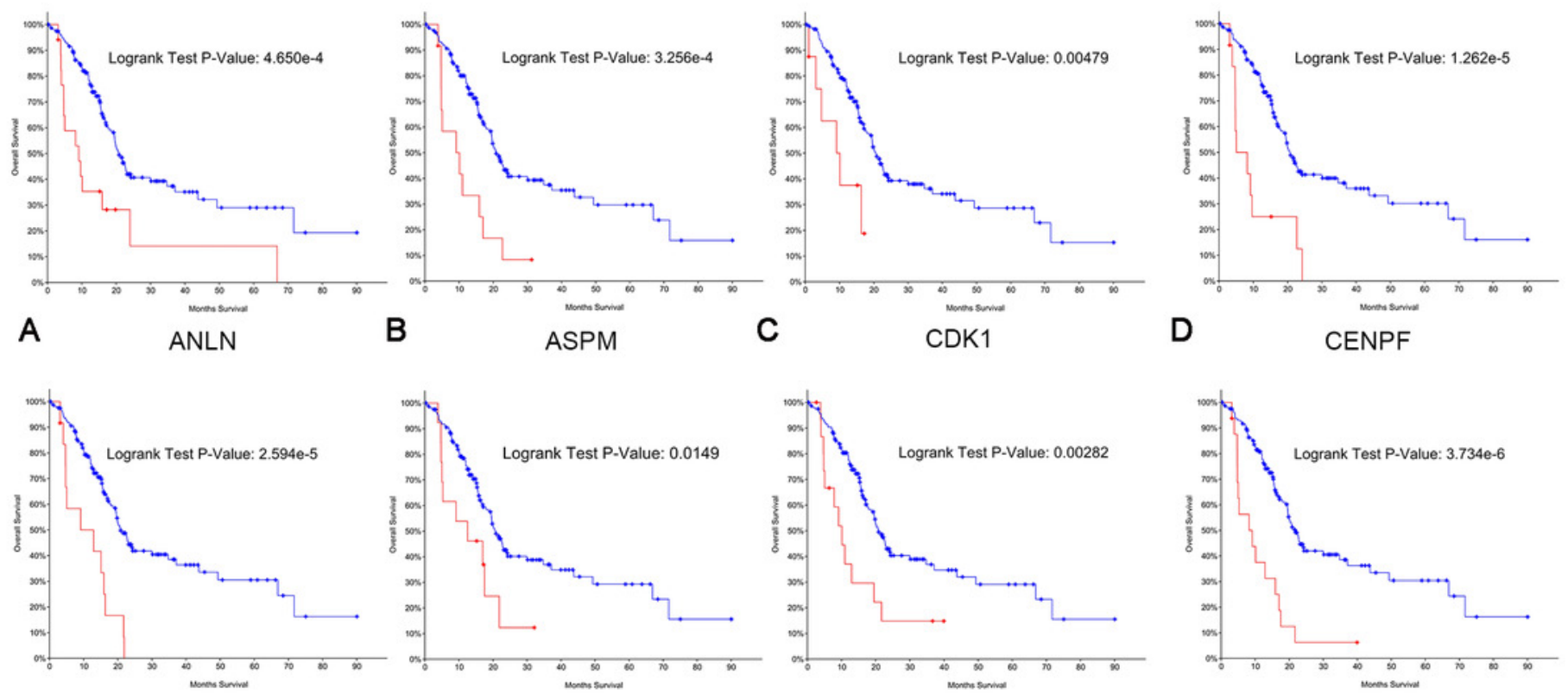

E

$\mathrm{F}$

G

ECT2

$\mathrm{H}$

NEK2

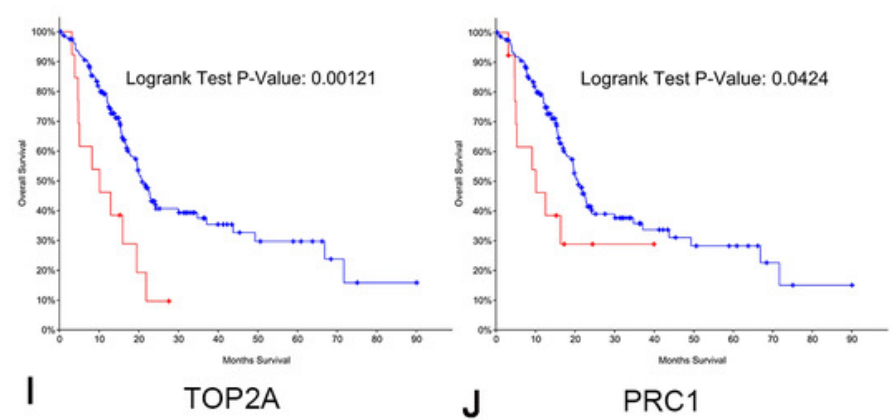




\section{Figure 6}

Disease-free survival analyses of hubgenes were performed using the cBioPortal online platform.

A log rank test $P<0.05$ was considered statistically significant.

- Cases with Alteration(s) in Query Gene(s)

- Cases without Alteration(s) in Query Gene(s)
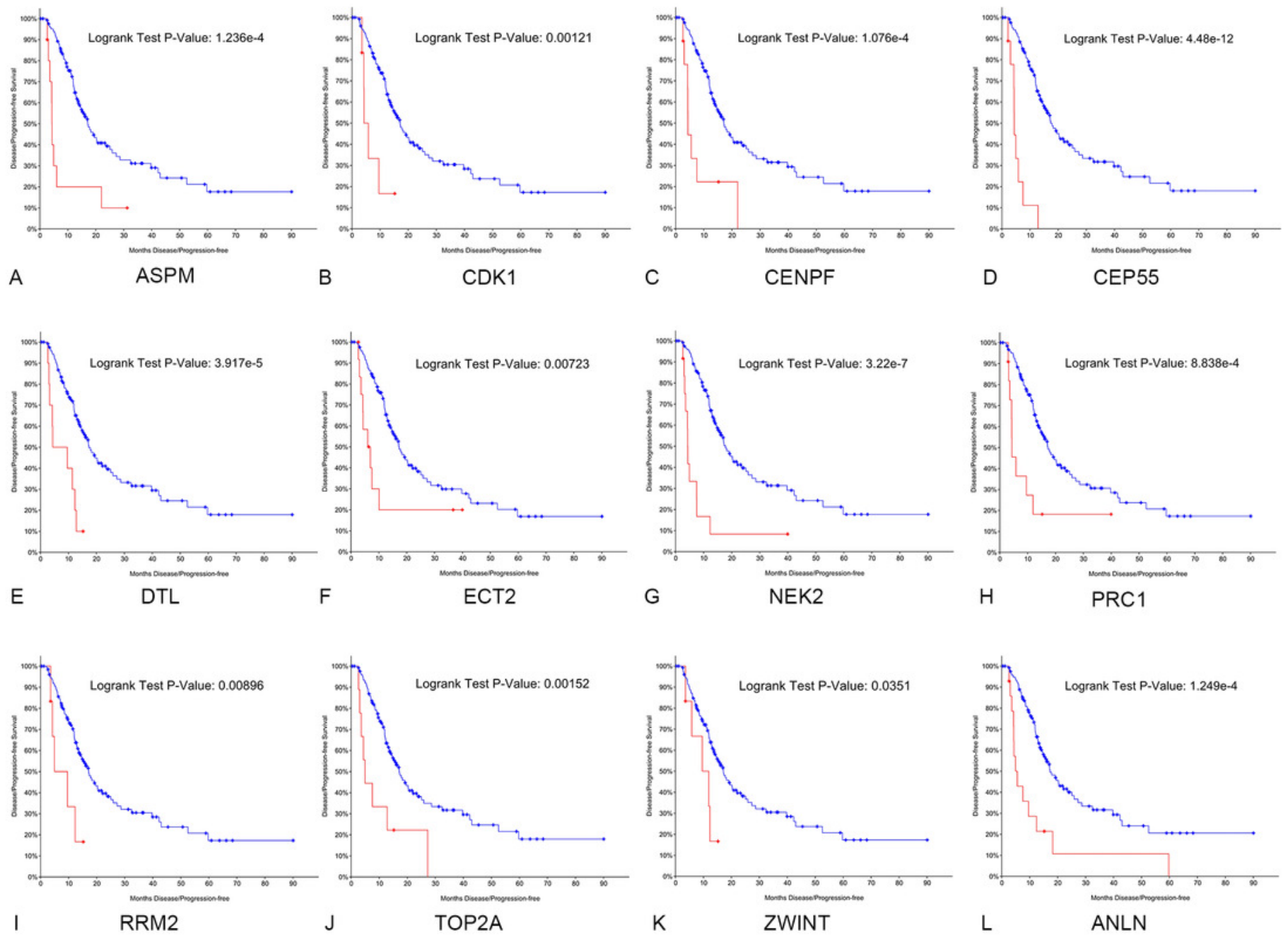


\section{Figure 7}

Oncomine analysis of (A) CDK1 and (B) CEP55 in cancer vs. normal tissue.

Heat maps of CDK1 and CEP55 gene expression in clinical pancreatic cancer samples vs. normal tissues. 1. Pancreatic Ductal Adenocarcinoma Epithelia vs. Normal Grutzmann Pancreas, Neoplasia, 2004. 2. Pancreatic Carcinoma vs. Normal Pei Pancreas, Cancer Cell, 2009. 3. Pancreatic Carcinoma vs. Normal Segara Pancreas, Clin Cancer Res, 2005. (C) CDK1 mRNA expression and (D) CEP55 mRNA expression in pancreatic cancer compared with normal pancreatic tissues in the Grutzmann Pancreas dataset. Association between the expression of (E) CDK1 and (F) CEP55 and tumor grade in the Grutzmann Pancreas dataset. P $<0.05$ was considered statistically significant. 
A $\begin{array}{rrr}\text { Median Rank } & \text { p-Value } & \text { Gene } \\ 337.0 & 1.37 E-7 & \text { CDK } 1\end{array}$

\begin{tabular}{|l|l|l|}
\hline 1 & 2 & 3 \\
\hline
\end{tabular}

1. $\mathrm{P}$-value $=1.37 \mathrm{E}-7$ fold change $=3.888$

2. $\mathrm{P}$-value $=0.006$ fold change $=4.942$

3. $\mathrm{P}$-value $=0.001 \quad$ fold change $=1.457$

$\stackrel{151025}{\square} \square \square \square \stackrel{25}{\square} \square \square^{5} \square^{1} \square \square$ Not measured

C

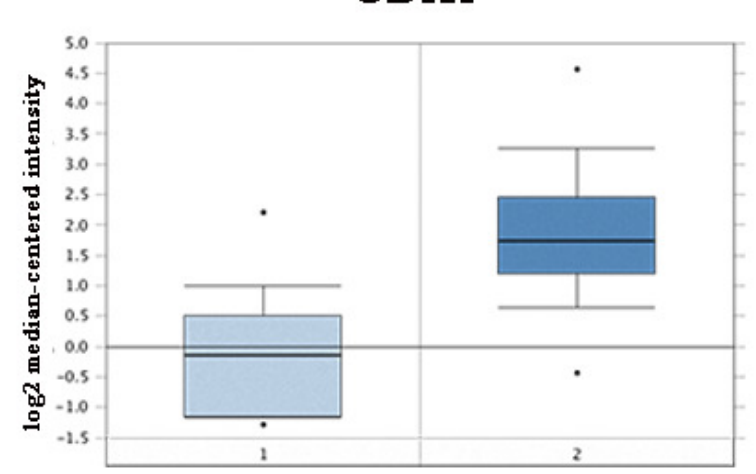

Legend

1. Pancreas (16)

2. Pancreatic Carcinoma (36)

E

CDK1 grade

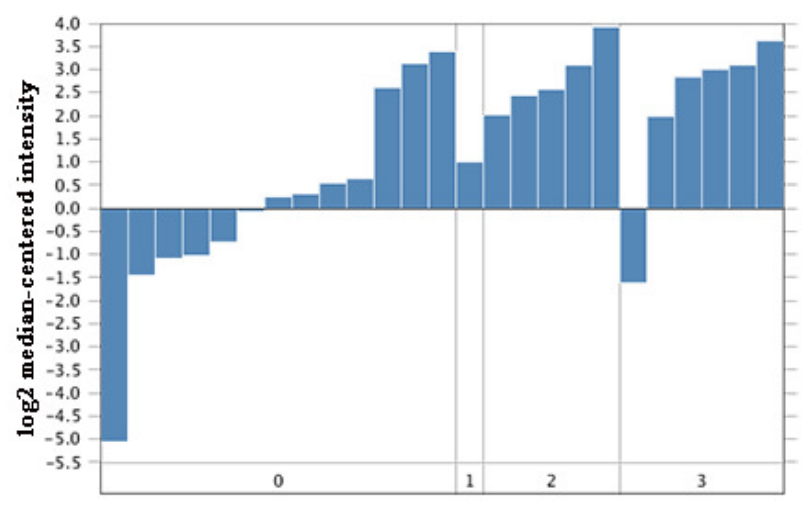

Legend

0 . No value (13) 1 .Grade 1 (1)

2.Grade 2 (5) $\quad 3$.Grade 3 (6)
B

\begin{tabular}{|c|c|c|c|c|c|}
\hline Median Rank & p-Value & Gene & & & \\
\hline \multirow[t]{2}{*}{87.0} & $6.13 \mathrm{E}-10$ & CEP55 & & & \\
\hline & & & 1 & 2 & 3 \\
\hline
\end{tabular}

1. $\mathrm{P}$-value $=6.13 \mathrm{E}-10$ fold change $=4.102$

2. $\mathrm{P}$-value $=3.61 \mathrm{E}-4$ fold change $=20.553$

3. P-value $=0.004 \quad$ fold change $=1.494$

D

CEP55

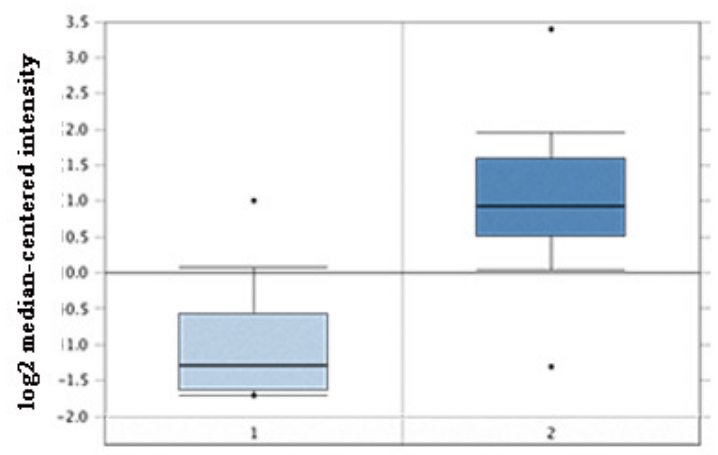

Legend

1. Pancreas (16)

2. Pancreatic Carcinoma (36)

F

CEP55 grade

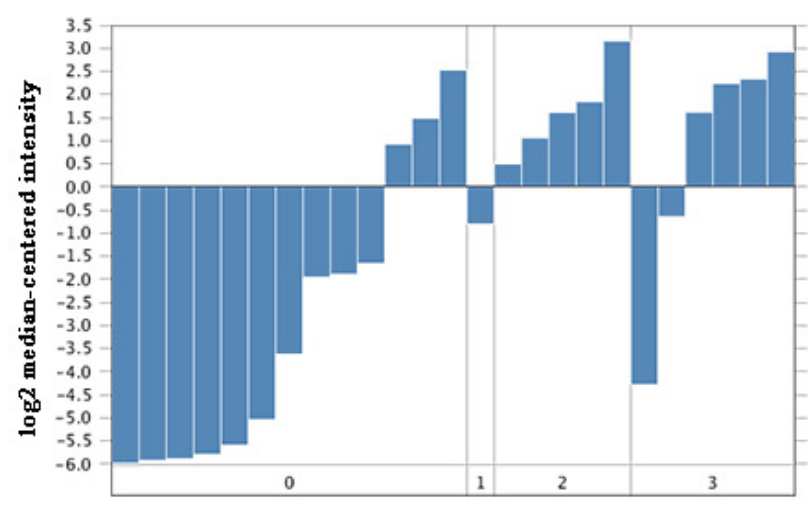

Legend

0 . No value (13) 1 .Grade 1 (1)

2.Grade 2 (5) 3.Grade 3 (6) 


\section{Figure 8}

The expression of CDK1 and CEP55 in clinical specimens.

(A, B) Western blotting detection of CDK1 and CEP55 protein in eight snap-frozen tumorous and tissues of pancreatic cancer patients. (C, D) The bar chart reveled that the expression ratio of CDK1 and CEP55 to GAPDH by densitometry. Data are represented as the mean \pm SEM (*P $<0.05$, tumor tissues compared with the non-cancerous adjacent tissues).

A

$\begin{array}{lllllll}\text { T1 } & \text { N1 } & \text { T2 } & \text { N2 } & \text { T3 } & \text { N3 } & \text { T4 }\end{array}$

CDK1

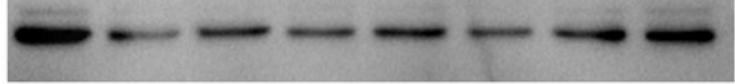

CEP55

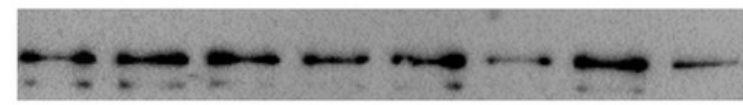

PCNA

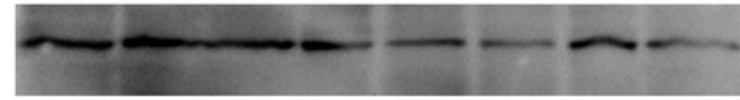

GAPDH

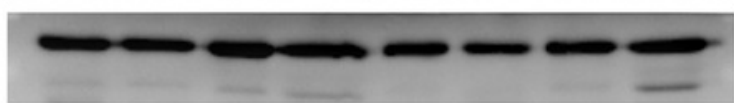

B



CDK1



CEP55

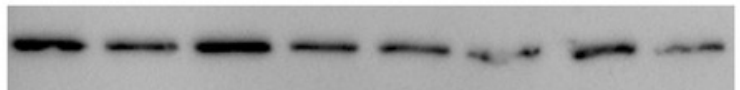

PCNA

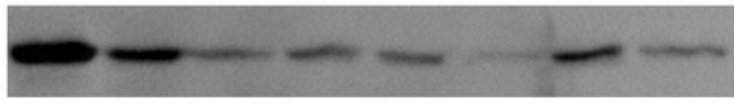

GAPDH
C



$\mathrm{D}$

CEP55

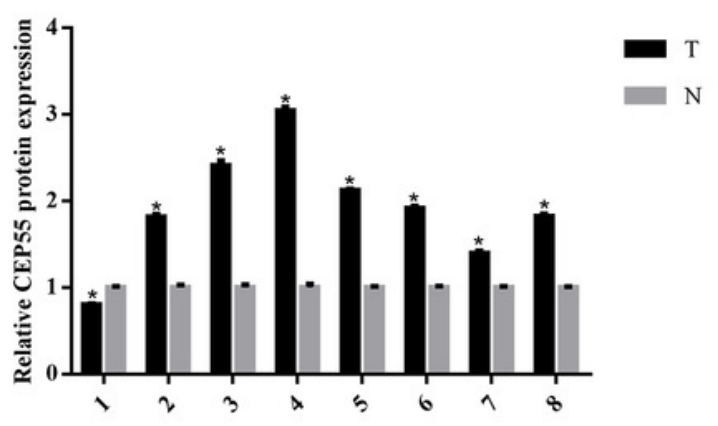

